\title{
SIMULATION MODELING FOR MILITARY LOGISTICS AND SUPPORTABILITY STUDIES
}

\author{
C. GREGORY JOHN \\ Midwest Modeling Technologies \\ P. O. BOX 6949 \\ ST. LOUIS, MO 63123
}

\author{
RODNEY E. SCHULTZ \\ McDonnell Douglas Missile Systems Company \\ MAILCODE 1063384
}

P. O. BOX 516
ST. LOUIS, MO 63166-0516

P. O. BOX 516
ST. LOUIS, MO 63166-0516

\begin{abstract}
Simulation has proven to be a valuable tool for McDonnell Douglas Missile Systems Company to make costeffective credible recommendations to the Government for a missile Serviceable-In-Service-Time (SIST). SIST is the period of calendar time that a missile may remain in operational use or storage before requiring recertification (a routine test and maintenance to assure the missile is operational) at a Naval Weapon Station (NWS) or Depot.
\end{abstract}

\section{THE TRADE-OFF}

To obtain the best SIST interval, both missile availability and cost must be considered. Missile availability is defined as the proportion of missiles estimated to be free of detectable failures that are available for operational use. To simplify this paper, cost will be limited to include the touch labor and repair parts to perform missile recertification and subsequent repair actions required to return the missile to Ready For Issue (RFI) status. A longer SIST interval implies a longer time between missile tests, which in turn implies a lower cost and a lower probability that a missile will be free of detectable failures. Figure 1 illustrates the theoretical point which provides the highest missile availability for a practical cost.

\subsection{Missile Availability}

Missile availability is a combination of asset readiness and reliability. It is calculated by

MISSILE AVAILABILITY = ASSET READINESS X RELIABILITY

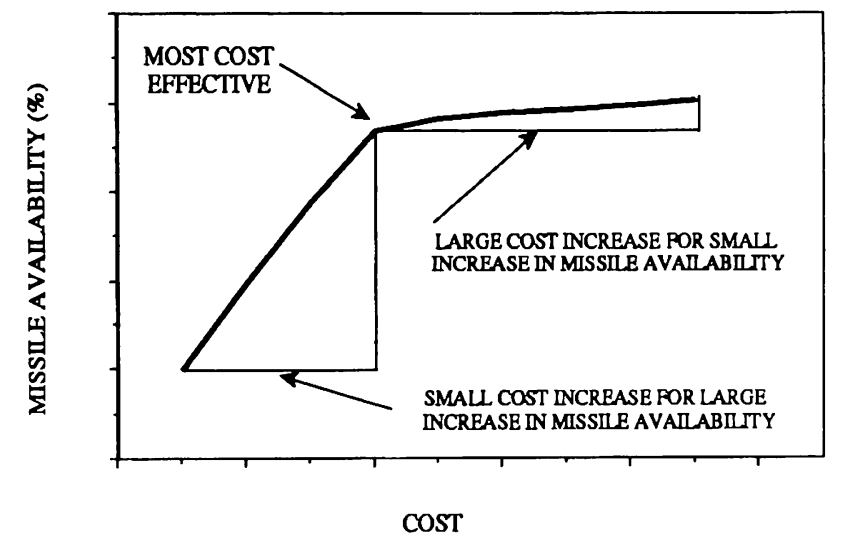

Figure 1: Highest Availability For Lowest Cost

A longer SIST interval will increase asset readiness but decrease reliability. The relationship between SIST intervals, missile availability, asset readiness, and reliability is shown in Figure 2.

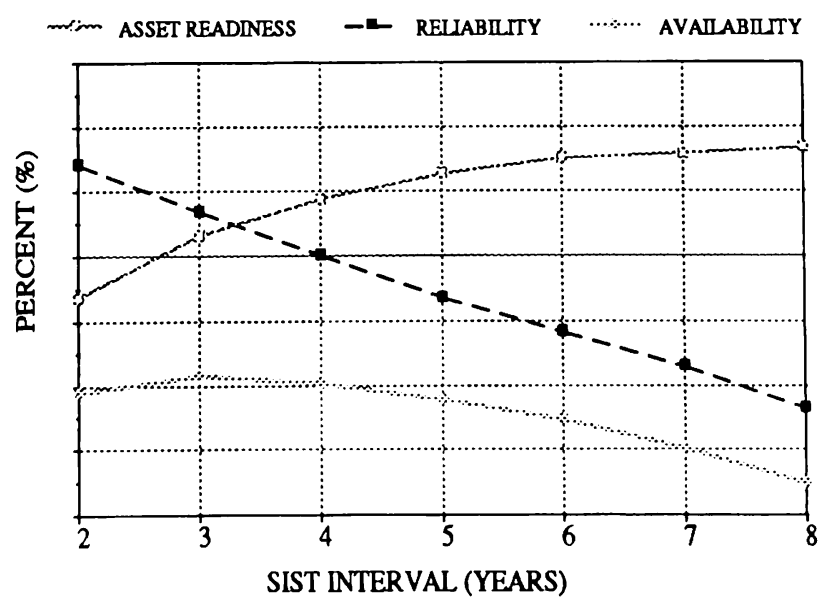

Figure 2: Missile Availability 
Asset readiness, as depicted in Figure 3 , is the proportion of the total missiles that are presumed to be operable (ie. capable of being put into immediate operation). The nonRFI items would be missiles undergoing maintenance and missiles that are in the pipeline to undergo maintenance or recertification. Another way of stating this is, asset readiness is the current quantity of RFI missiles divided by the current quantity of missiles in the inventory (RFI + non-RFI).

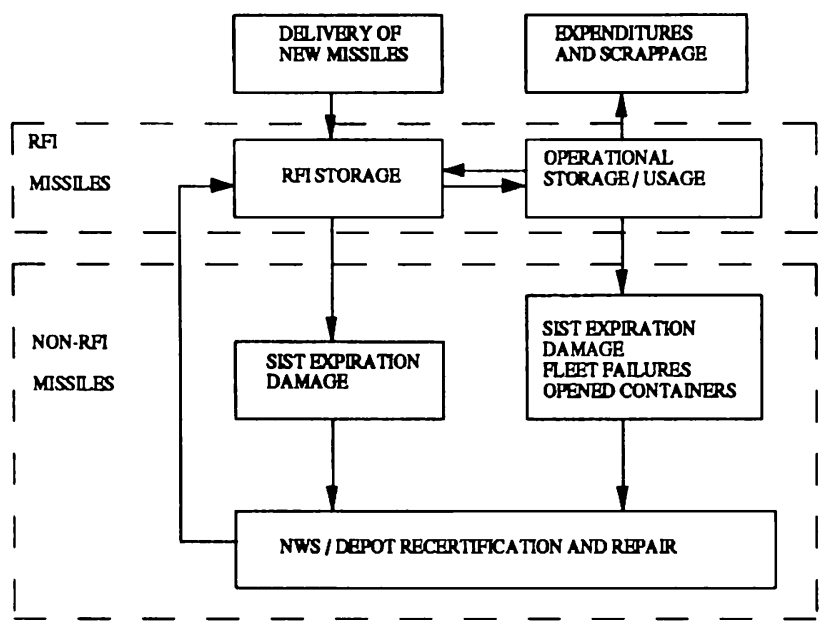

Figure 3: Asset Readiness

Asset readiness is directly related to the length of the SIST. Greater asset readiness would be obtained by having a longer SIST interval. A longer SIST interval means that fewer missiles require routine recertification tests. Thus asset readiness increases as shown in Figure 2.

Reliability is the probability an RFI missile will successfully perform its mission. Reliability estimates are based upon Mean Time Between Failure (MTBF) and elapsed time in each environment since the last recertification. The environments include conditions such as missiles stored in containers, missiles out of containers in a ready service condition, and missiles on an aircraft in captive carry.

Reliability is inversely related to the length of the SIST. A greater reliability would be obtained by having a shorter SIST interval. Since the reliability of a system decreases over time, a shorter SIST interval means shorter intervals between recertification tests and greater reliability, as shown in Figure 2.

In summary, Figure 2 illustrates that a longer SIST interval results in higher asset readiness but lower reliability, while a shorter SIST interval results in lower asset readiness but higher reliability. Thus, good estimates of each of these measures are necessary to study their combined effect on missile availability.

\subsection{Cost}

Cost is another vital factor in determining the best SIST interval for a cost effective missile availability. Cost estimates for this paper were partial costs and used only to determine relative cost impacts, not actual costs. All costs are fixed year costs with no inflation factor. The costs for this paper include the touch labor required to perform a missile recertification and average touch labor and repair parts required to repair the failed missiles and their associated subassemblies.

Cost is inversely related to the length of the SIST. The lower cost would be obtained by having a longer SIST interval. Longer SIST intervals result in fewer tests and lower cost as shown in Figure 4.

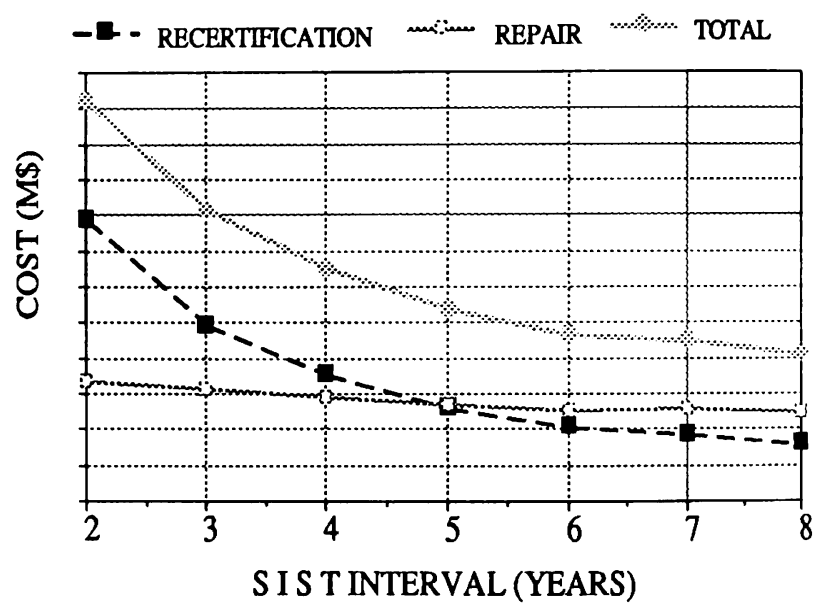

Figure 4: Increased SIST Reduces Cost

\section{METHODOLOGY}

Simulation modeling was chosen to analyze this complex relationship among SIST, cost, and missile availability in the supportability process because of its flexibility and its capability to accurately analyze complex processes.

Other methodologies using different versions of analytical math models were investigated. These models are good tools for analyzing relatively simple processes where mathematical approximations can be developed to represent the process. However, many of the areas studied had interdependencies and interaction between the processes. These interdependencies are very difficult (and sometimes impossible) to represent in a math model. With simulation it is possible to analyze these complexities because the actual flows and decision making processes are modeled instead of relying on simplified mathematical representations of the processes. 
Animation of the simulation process was also used effectively to visually depict the process flow and interactions. This feature helped in the understanding of model relationships. It was used during the model verification and validation phases to troubleshoot problem areas and to help assure that the model was performing as expected and providing credible results. In addition, animation was a great communication tool to explain to others how the simulation model performed, in order to receive their comments and assistance.

\subsection{Simulation Model}

This model is a discrete event simulation model written in the SIMAN simulation language, augmented with FORTRANevent subroutines. The CINEMA software package wasused to develop the animations of the simulation model.

The supportability model includes the entire life cycle of the missile as depicted in Figure 5. Beginning with new build missiles leaving the factory, the model includes transportation to the NWS, storage at the NWS, deployment to ships and forward sites, missile expenditures, returns to the NWS for recertification or field failure, and transportation between the NWS and repair depots.

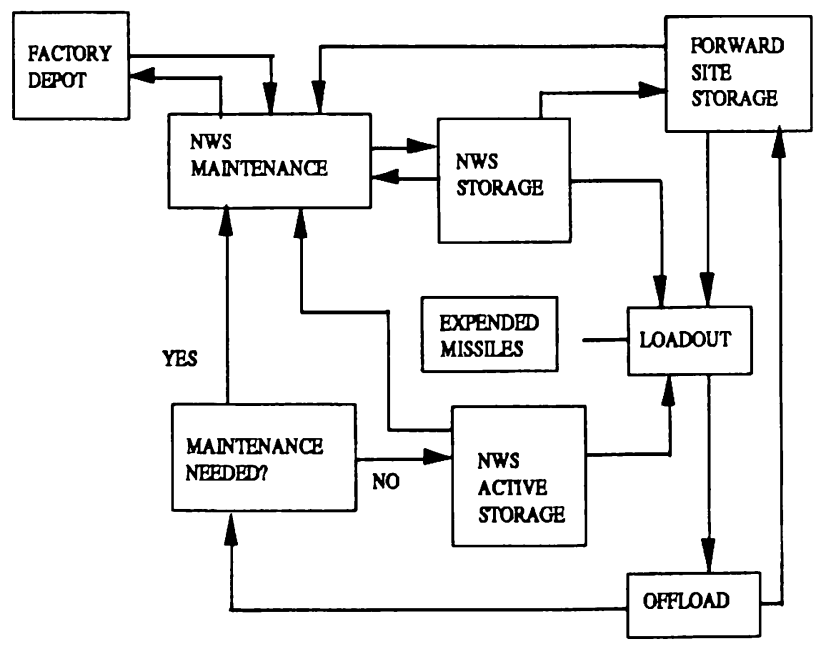

Figure 5: Entire Supportability Process Modeled

\subsection{Assumptions}

The supportability simulation model is capable of modeling a variety of assumptions regarding production, delivery, storage, and operational scenarios. The relevant assumptions used for this paper are described in the following paragraphs.

The model is designed to handle a large quantity of missiles. Quantities of 15,000 to 20,000 missiles produced over a 5 to 10 year period are typical.
The system is usually simulated for a period of thirty years, starting with the first year of production. Missiles are transported by truck from the factory to the Naval Weapon Stations, with a percentage allocation to each fleet. After transportation and administrative delays, the missiles are placed in storage until they are deployed or until the SIST interval requires a recertification.

The operational scenario includes a wide missile deployment profile by fleet including several different using activities such as aircraft carriers, amphibious assault ships, Naval Air Stations, and Marine Corps Air Stations. Each of these activities are modeled independently with different initial start dates, individual scenarios, quantities of allocated and ready service missiles. Fleet training exercises are also modeled to account for missiles being expended.

The carriers and amphibious assault ships are scheduled to go on cruises lasting approximately six months, followed by approximately twelve months spent in port. The cruises are scheduled so that there is a minimum and maximum number of ships at sea at any point in time. Missiles to be deployed from storage are chosen at random, with the condition that no missile will be deployed with less than nine months SIST remaining.

Ready service missiles are those missiles which have been removed from their shipping containers and are available for immediate usage. The ready service missiles are modeled on a number of rotations per cruise, consisting of variable hours below deck, above deck, and a captive carry mission. Missiles are tested only at the beginning and end of a captive carry mission. Any missile failing test is sent below deck and replaced in ready service by a missile from the ship's RFI magazine. At the end of a cruise, any missile that has failed test, been in ready service, or has less than nine months of SIST remaining is sent back to the NWS for recertification. Other missiles are placed in active storage (available for cross-decking to another ship), and these missiles will be given priority to go on subsequent cruises before missiles will be removed from deep storage at the Naval Weapon Station.

Failed missiles are detected only when a missile is loaded on an aircraft and tested, or when a missile is tested during recertification at the NWS. In either case, the simulated test results are based upon the amount of time the missile has spent in each of several environments with different MTBF estimates for each environment. Test thoroughness and confirmation factors are applied to the different levels of testing.

There is a monthly check of missiles in storage to see if their SIST interval has expired. If any have, they are removed from storage and recertified. Altemative scenarios have been examined employing sampling strategies to determine which missiles to remove from storage for recertification. 
Missiles returned to the NWS for recertification or repair receive a comprehensive check out prior to being placed back into a RFI status. Then it is placed back into storage.

\section{ANALYSIS}

Simulation model outputs have been developed to provide the key information required to make conclusions. Model outputs have been modified as the simulation model matured and additional uses of the model outputs were developed. The output reports are available in two types. The first consists of standard reports that are available each time the simulation model is run. The second type are customized reports that are specifically designed to meet a certain requirement and may only be required for a single analysis. In addition, to supplement these reports, data files of raw detailed information can be created in order to conduct detailed ad hoc analysis and investigation.

The key information from both standard and customized reports are then summarized and presented utilizing conventional business graphics for ease of comparative analysis. The quantity of detailed data available from the simulation model and the flexibility for data manipulation has been extremely useful in providing the right comparative analytical values upon which our concurrent engineering teams can draw conclusions and make decisions.

\section{RESULTS}

\subsection{Studies Performed}

A systematic approach was used to analyze the many different variables that could affect the determination of the optimal SIST. The results from the model may not recommend the optimal SIST, but will provide a near optimal recommendation based upon the information that is available for that phase of the program.

A baseline condition is selected and then deviations from this baseline are analyzed to evaluate either improvement or degradation in the process. Even if the baseline condition may not represent the actual parameters when a missile design is finalized and the missile deployed, the model results will still be relative. These relative results can be used to accurately analyze impacts and trends for comparative analysis.

\subsection{Study Results}

The SIST study analyzed the impacts of different values of the SIST interval. The baseline interval for the SIST was selected to be 5 years. SIST intervals of $2,3,4$, 6,7 and 8 years were also analyzed to determine the impact of SIST on system performance measures.
Figure 6 shows that as the SIST interval increases, the total quantity of recertifications decreases. The missiles failing test in the fleet and those with opened containers represent a small proportion of the total recertifications and are not significantly impacted by changes in the SIST. The significant changes in recertifications are those missiles coming from either storage or active storage.

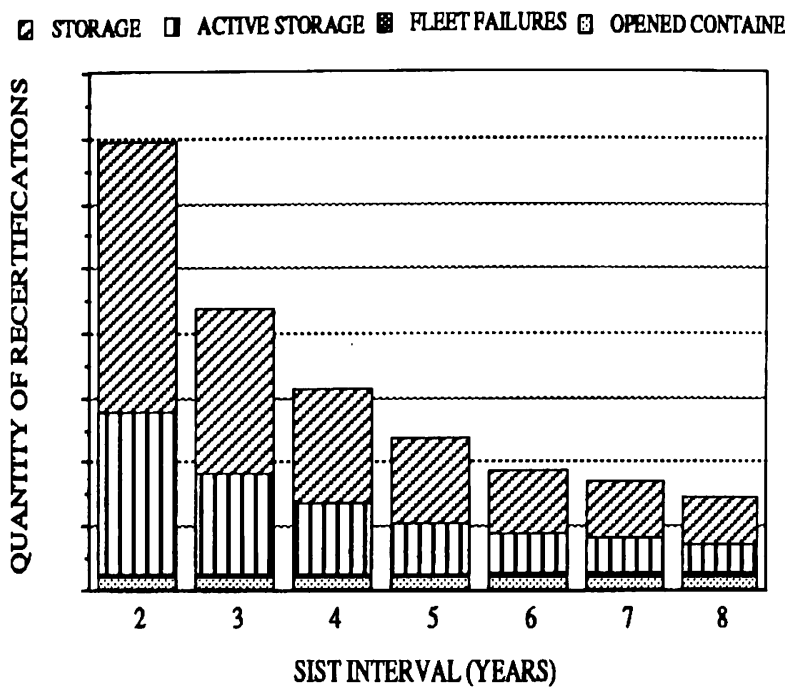

Figure 6: Increased SIST Reduces Recertifications

Figure 7 shows that as the SIST interval increases the total number of failed missiles also decreases. However the downward trend is not as dramatic as it was for recertifications.

G STORAGE $\square$ ACTIVE STORAGE FLEETPAILS 8 OPENED CONTANERS

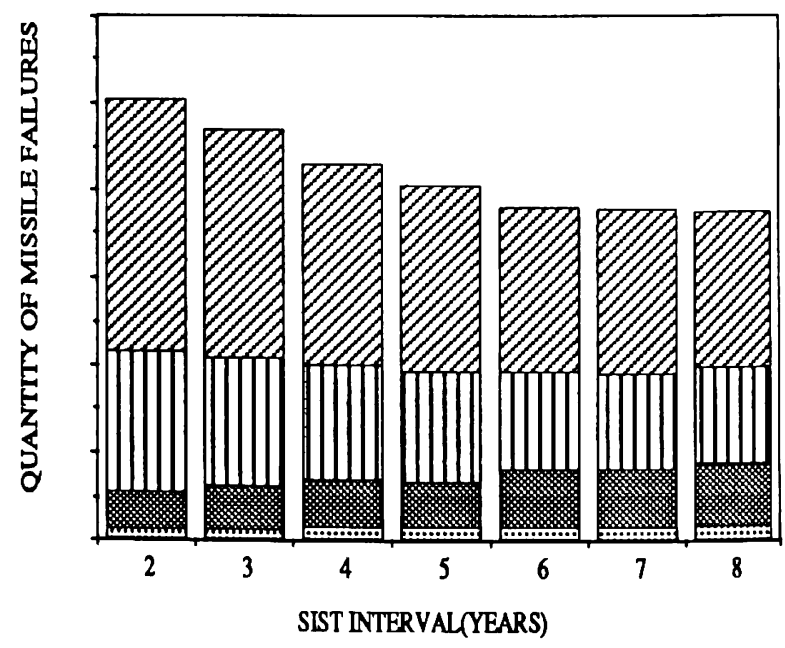

Figure 7: Increased SIST Reduces Missile Failures

Significant cost savings can be obtained by increasing the SIST interval as shown in Figure 8. The biggest savings will be in the recertification cost of good missiles, with only minor savings in total repair cost. 


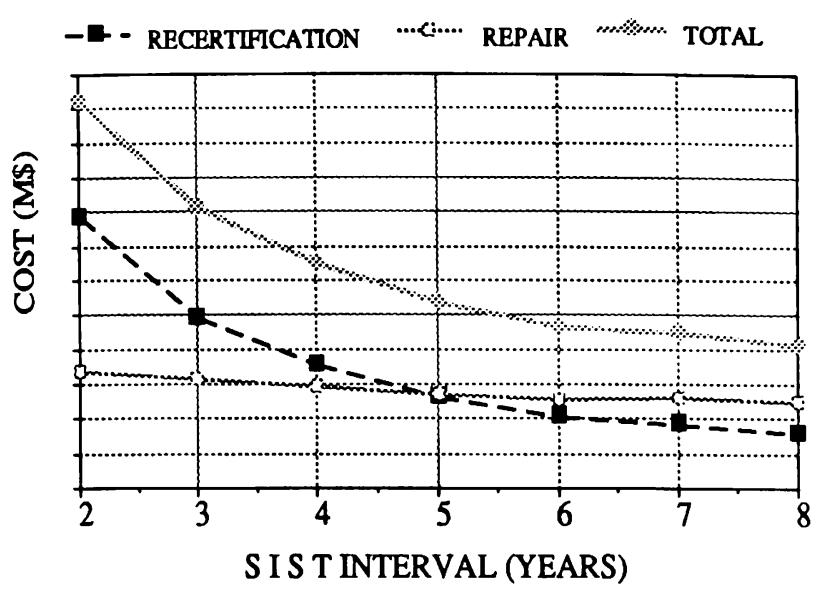

Figure 8: Increased SIST Reduces Total Cost

Figure 9 shows that as the SIST interval increases, the missile asset readiness improves while the reliability decreases. The highest availability without regard to cost is between a 3 and 4 year SIST.

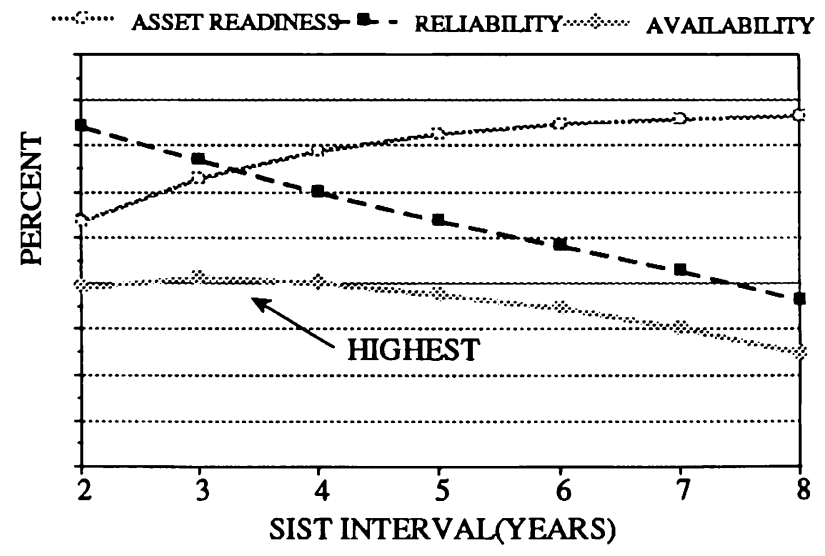

Figure 9: 3-4 Year SIST Provides Highest Availability

However, when cost is considered, a 5 year SIST yields the most cost effective missile availability as shown in Figure 10. For example, reducing the SIST interval from 7 years to 6 years or 6 years to 5 years has a significant increase in missile availability with only a small increase in cost. However, it is not cost effective to go from a 5 year SIST to a 4 year SIST because there is only a small increase in missile availability, with a somewhat larger increase in cost. Therefore based on having the largest quantity of good missiles in the fleet for the lowest practical cost, the model would recommend a 5 year SIST.

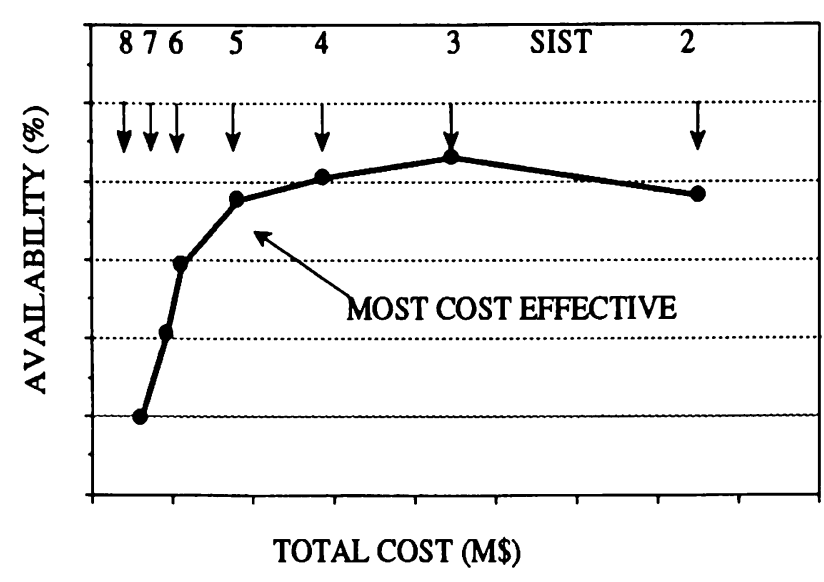

Figure 10: 5 Year SIST Most Cost Effective

\subsection{Sensitivity Studies Performed}

Although this paper has concentrated on the methodology for determining the most cost effective SIST, the intended purpose of the model was to analyze the entire supportability process with its complexities and interrelationships. Studies have been performed to evaluate many of the system parameters which are the major contributing factors to the supportability process. A few of the other factors analyzed which impacted the determination of the most cost effective SIST were:

1. Variations in the MTBF

2. Sampling concepts for recertifying missiles in storage

3. Quantity of ready service missiles

4. Length of time that an individual missile should remain in ready service condition

5. Location and frequency to perform fleet testing of the RFI missiles

6. Impacts of ongoing missile improvement programs

7. Variations in processing times

\subsection{Conclusions}

The supportability simulation model has proven to be a very useful and effective concurrent engineering tool. It has been able to provide the detailed information required in the format requested. The model has been validated, and is providing credible results. Through the use of the simulation model, credible cost effective SIST recommendations have been made to the customer. 


\section{AUTHOR BIOGRAPHIES}

C. GREGORY JOHN is a partner in Midwest Modeling Technologies of St. Louis, Missouri. He holds a Ph. D. and M. S. in Management Science from the Pennsylvania State University, a B.S. in Business and a B. A. in Mathematics from the University of Kansas. He has served on the faculty at both Penn State and Indiana University. His interests include the application of optimization and simulation techniques to problems in operations management and logistics.

RODNEY E.SCHULTZ is a Staff Specialistat McDonnell Douglas Missile Systems Company in St. Louis, Missouri. He received a B. A. in Mathematics from Washington State University and an M. S. in Statistics from the University of Arizona. His current interests include simulation modeling of production and military logistics for new missile programs in the development stages. 